\title{
Predictions of Skin Permeability Using Molecular Dynamics Simulation from Two-Dimensional Sampling of Spatial and Alchemical Perturbation Reaction Coordinates
}

\author{
Magnus Lundborg $^{1, \bowtie}$, Christian Wennberg $^{1}$, Jack Lidmar ${ }^{2}$, Berk Hess ${ }^{3}$, Erik Lindahl ${ }^{4,5}$, and Lars Norlén ${ }^{6,7, \bowtie}$ \\ ${ }^{1}$ ERCO Pharma AB, Science for Life Laboratory, Solna, Sweden \\ ${ }^{2}$ Department of Physics, KTH Royal Institute of Technology, Stockholm, Sweden \\ ${ }^{3}$ Department of Applied Physics, Science for Life Laboratory, KTH Royal Institute of Technology, Solna, Sweden \\ ${ }^{4}$ Department of Biophysics and Biochemistry, Science for Life Laboratory, Stockholm University, Solna, Sweden \\ ${ }^{5}$ Department of Applied Physics, Swedish e-Science Research Center, KTH Royal Institute of Technology, Stockholm, Sweden \\ ${ }^{6}$ Department of Cell and Molecular Biology (CMB), Karolinska Institutet, Stockholm, Sweden \\ ${ }^{7}$ Dermatology Clinic, Karolinska University Hospital, Stockholm, Sweden
}

\begin{abstract}
A molecular level understanding of skin permeation may rationalize and streamline product development, and improve quality and control, of transdermal and topical drug delivery systems. It may also facilitate toxicity and safety assessment of cosmetics, skin care products. Here, we present new molecular dynamics simulation approaches that make it possible to efficiently sample the free energy and local diffusion coefficient across the skin's barrier structure and predict skin permeability and the effects of chemical penetration enhancers. In particular, we introduce a new approach to use two-dimensional reaction coordinates with so-called Accelerated Weight Histograms, where we combine sampling along spatial coordinates with an alchemical perturbation virtual coordinate. We present predicted properties for twenty permeants, and demonstrate how our approach improves correlation with ex vivo/in vitro skin permeation data. For the compounds included in this study, the obtained $\log \mathrm{K}_{\mathrm{P}_{\text {exp-calc }}}$ mean square difference was $0.8 \mathrm{~cm}^{2} \mathrm{~h}^{-2}$.
\end{abstract}

Skin permeability | Transdermal delivery | Molecular dynamics simulation | GROMACS | Enhanced sampling | Accelerated Weight Histogram method | Alchemical perturbations

Correspondence: magnus.lundborg@ercopharma.com and lars.norlen@ki.se

\section{Introduction}

Transdermal and topical drug delivery allows for noninvasive, pain-free, continuous drug administration with reduced side effects and increased patient compliance compared to per oral or intravenous drug delivery.

Assessment of skin permeability is important for the design of percutaneous drug delivery systems. Today, the dominating means of investigating drug skin permeability and the effects thereon of different delivery vehicle excipients is by $e x$ vivo/in vitro testing. There are, however, ethical problems involved in the study of percutaneous drug delivery using excised human or animal skin, or using animals in vivo. $(1,2)$ There are also issues of the translation of animal drug delivery data to humans.(1-3) When using human skin, there are complications of inter- and intraindividual variation as well as of inter-laboratory differences. $(1,4)$ Furthermore, ex vivo/in vitro testing yields no information about how compounds permeate skin.

In silico modeling is an interesting alternative to predict skin permeability and to gain insights into how compounds are absorbed in skin (5), but it requires both faithful models of the skin system and methods that can sample the process efficiently. A skin permeation prediction model relevant for human skin, encompassing the effects of penetration enhancers and other drug delivery vehicle excipients, could help improve drug delivery formulation design.

We have previously shown that an atomistic model of the skin's barrier structure, i.e., the intercellular lipid matrix of the stratum corneum, validated against cryo-electron microscopy (cryo-EM) data from near-native skin (6) (Fig. 1), could be used in molecular dynamics (MD) simulations to predict the permeability of molecules through skin (7). These models made it possible to reproduce the effects of chemical penetration enhancers on the skin's barrier structure at least qualitatively (7), which enabled a molecular-level understanding of their mechanisms of action, but the amount of sampling required and limited convergence made it difficult to accurately match experimental values. Improving the efficiency of sampling for this type of complex system has led to a number of improvements of simulation protocols, in particular with respect to the sampling of the free energy landscape and local diffusion coefficient of drugs passing through the skin's barrier structure (8).

We here apply the Accelerated Weight Histogram (AWH) method, using reaction coordinates that combine a spatial dimension, pulling the molecule across the barrier structure, with a second alchemical free energy dimension. (8) This has made it possible to sample the free energy landscape and local diffusion coefficient of 20 chemical compounds with molar masses ranging from $18 \mathrm{~g} \mathrm{~mol}^{-1}$ to $\sim 300 \mathrm{~g} \mathrm{~mol}^{-1}$ and $\log \mathrm{P}_{\mathrm{o} / \mathrm{w}}$ from -2.1 to 4.6 . It is expected that such sampling, allowing decoupling of the permeant, will be efficient (9) in systems with long correlation times, such as the skin's barrier structure. An improved agreement between calculated and 
measured permeability coefficients is presented. It is shown that, using the AWH method, ex vivo/in vitro measured skin permeability data can be accurately predicted, even though the in silico model selectively accounts for the skin's barrier structure and not for the complete skin.

Molecular dynamics skin permeation modeling. Quantitative Structure-Permeability Relationship (QSPR) methods (10-12) are commonly used to predict skin permeability coefficients. Molecular dynamics (MD) simulations constitute an alternative and/or complement to QSPR methods. MD simulations are significantly slower than QSPR methods, but not as limited to a specific applicability domain. Further, incorporating the effect of formulation excipients on the permeability of active pharmaceutical ingredients (APIs) requires retraining of QSPR models, preferably for each combination of excipients. There have, nevertheless, been QSAR (Quantitative Structure-Activity Relationship) (13) studies involving chemical permeation enhancers, but information about these excipients' mechanisms of action has been difficult to obtain. With MD simulations, formulation excipients can be incorporated in the skin's barrier structure in their most probable concentrations, locations and orientations, based on their local free energy potentials. This enables studying how they interact with the barrier and how they affect the permeability of one or more APIs.

One limitation of the MD simulation approach presented here is that only the structure of the main permeability barrier in skin $(14,15)$, and not the complete skin, is studied in detail. However, it would be possible to complement the calculated permeability coefficients with mathematical models accounting for corneocyte permeability as well as for diffusion through the viable epidermis and dermis. $(16,17)$

In our previous studies we showed that it is possible to predict the permeability coefficient of different chemical compounds through an atomistic model of the skin's barrier structure (6, 7), illustrated in Fig. 1. Therein we used MD simulations to pull the permeants through the barrier system using a stiff spring and the forward-reverse (FR) method to calculate the permeability coefficients.(18-21)

Based on our previous proof-of-concept study (7) we have studied the effects of the pulling speed on the calculated permeability coefficients in more detail. In the supplementary material (Fig. S1) we illustrate sampling problems using potentials of mean force (PMFs), i.e., the relative free energy difference across the system, of testosterone that we have experienced to be a permeant that needs careful sampling for obtaining accurate results. The general observation was that with slower pulling speeds the PMFs grew more detailed and the result free energy barriers became lower. We attribute this to the skin's barrier lipid system being in a gellike state and that the permeant needs to be pulled slowly to remain in a state near equilibrium, even if forward-reverse pulling is a nonequilibrium method (20). The experience that slower pulling speeds resulted in lower PMFs correlated in time with the publication by Wang and Klauda (22), in which they stated that all 80 (approximately) stacked lipid bilayers of the skin's barrier structure must be taken into ac- count (22-24), since the calculated permeability coefficient is not actually an average speed through the system, even if its unit would indicate that. The effect is that the permeability coefficient should be divided by 80 , or 1.9 be subtracted from its $\log$ value. When taking 80 stacked lipid bilayers layers into account for calculating the permeability coefficients using the data from our previous study(7) the $\log \mathrm{K}_{\mathrm{P}_{\text {exp-calc }}}$ mean square difference was approximately $\sim 11 \mathrm{~cm}^{2} \mathrm{~h}^{-2}$ (cf. $\sim 3 \mathrm{~cm}^{2} \mathrm{~h}^{-2}$ in the original work(7)). This made us conclude that very slow pulling speeds might be required to obtain correct PMFs. This would, in turn, require very long simulation times for each pulling simulation (see Figs. S1 and S3) - potentially so long that entirely different sampling approaches are required to predict permeability with reasonable amounts of computing time. Since it is not the main topic of this paper we refer to the supplementary information for more discussions about FR pulling and umbrella simulations.

A permeant's skin permeability coefficient is mainly determined by the height of the peaks, relative to the depth of the troughs, of its potential of mean force (PMF), i.e., the local free energy across the skin's barrier structure, assuming that the passage through the barrier structure is the rate limiting step for diffusion. On the other hand, the partitioning of a permeant from a formulation into the skin's barrier structure is primarily depending on the depth of the troughs of the permeant's PMF. It is therefore important that the MD simulations can reproduce the whole PMF as accurately as possible, given the available computational resources.

\section{Skin permeability calculations with the Accelerated Weight Histogram method. The Accelerated Weight His-} togram $(\mathrm{AWH})$ method $(25,26)$ is an extended ensemble technique, in which an adaptive bias is used to flatten the free energy landscape. The applied bias enables sampling high free energy (low probability) configurations to the same extent as low free energy states. It is also possible to customize the target sample distribution to focus more on regions of the free energy landscape that are of higher interest.

In the work presented here we have used the AWH method to improve the sampling of the permeability of 20 permeants through the skin's barrier system. Since GROMACS 2021 it is possible to use a two-dimensional AWH sampling with an alchemical free energy dimension (8) combined with a spatial dimension, i.e., pulling the permeant across the skin's barrier system. The alchemical free energy calculations imply that the interactions of the permeant with its surroundings can be gradually decoupled (switched on or off), thereby estimating the free energy of insertion. This means that there is no need for separate calibration of the PMFs in relation to the delivery vehicle, as all points along the PMF will be calibrated compared to a vacuum state, in the same way as the solvation free energies are calculated. This also improves the sampling in regions with very slow diffusion (long correlation times), since turning off the interactions with the surroundings will let the permeant leave that region in order to sample other parts of the landscape, and later return in a different configuration.(9) Fig. 2 shows a general overview of 


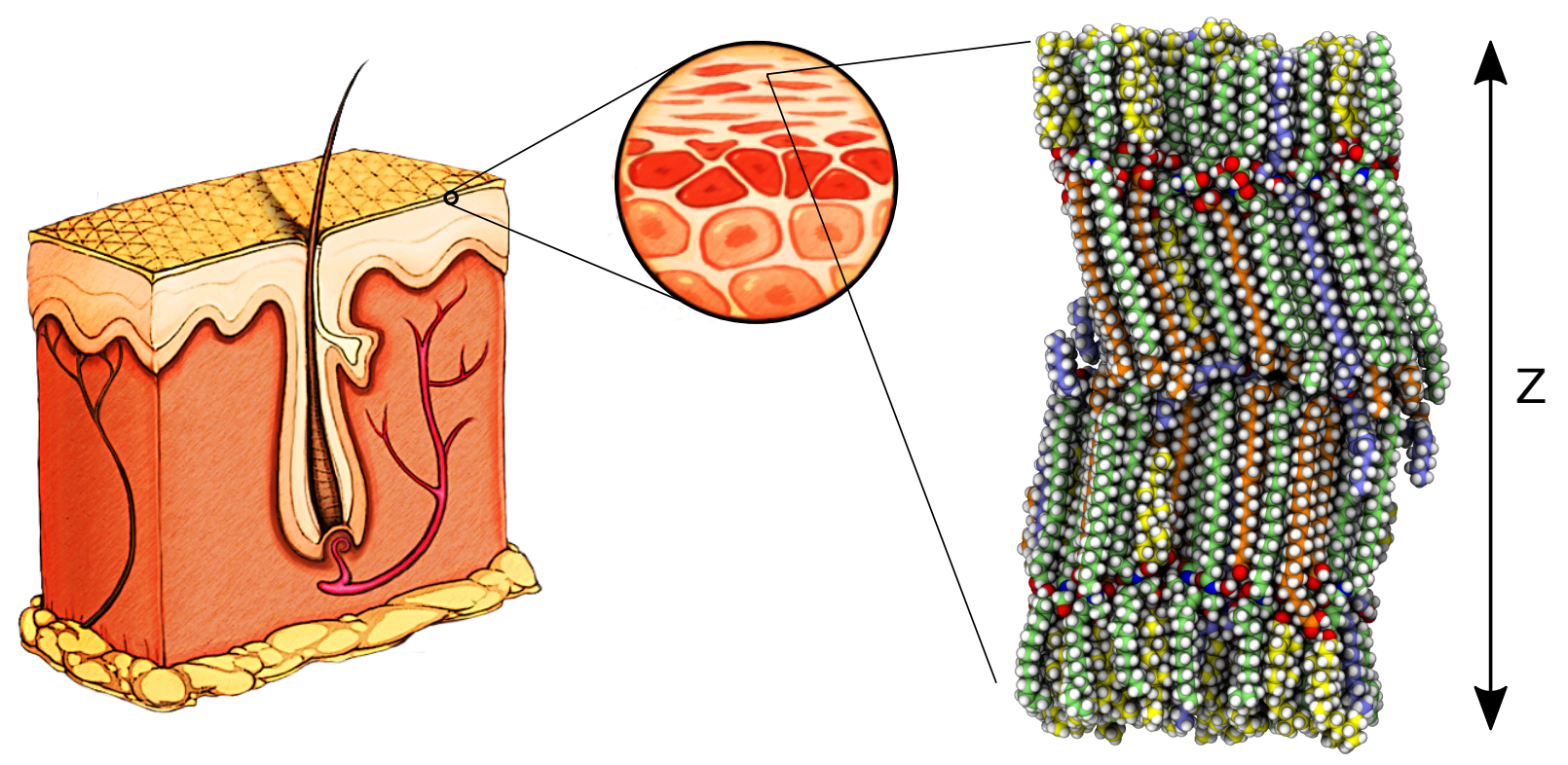

Fig. 1. A schematic representation of the structure of epidermis and dermis. To the right is shown a snapshot of the atomistic model of the intercellular lipid structure of human stratum corneum that constitutes the skin's main permeability barrier.(6) The arrow indicates the main permeability direction, also referred to as the $Z$ dimension. In the MD simulations the system is in practice repeated infinitely in the three dimensions. The carbon atoms are coloured based on the molecule type, where ceramides are green, acyl ceramides (ceramide EOS) are light blue, free fatty acids are orange and cholesterols are yellow. Hydrogen, nitrogen and oxygen atoms are colored white, blue and red, respectively.

the method applied to calculating the PMF of testosterone through the skin's barrier structure. With the AWH method it is also trivial to extend the simulations until they are sufficiently converged. This solves the primary sampling problem from previous studies, and it also enables us to calculate the local diffusion coefficient based on the AWH friction metric along the spatial dimension. The free energy of solvation in the solvent, or formulation, should still be calculated, which can be done in a separate one-dimensional alchemical AWH simulation.(8)

\section{Calculating permeability coefficients as a two-step} process. In simulation systems where the delivery vehicle, or solvent, is present along with the permeability barrier it is common to calibrate the PMF by setting it to zero in the delivery vehicle.(27) If the delivery vehicle is not simulated, like in the case of the stacked bilayer system studied here, the PMF can be calibrated based on a separate solvation free energy calculation.(7) However, once the permeant has partitioned from water into the barrier structure it is the relative free energy barriers that are the important factor, with troughs in the PMF acting as traps. Shifting the PMF so that its minimum is 0 is similar to what Wang and Klauda (22), Venable et al. (28) have done before.

In this project we have used a two-step process for the analysis of the permeability. The PMF, for calculating the permeation resistance, of one layer was calibrated relative to the hydration free energy. The permeation resistance through the remaining 79 layers was calculated with the PMF minimum set to 0 .

Applying this two-step permeability process, as well as accounting for the 80 stacked bilayers (described above), changed the $\log \mathrm{K}_{\mathrm{P}_{\text {exp-calc }}}$ mean square deviation in our proofof-concept study (7) from $\sim 3 \mathrm{~cm}^{2} \mathrm{~h}^{-2}$ (using the reference data in the original article(7)) to $\sim 17 \mathrm{~cm}^{2} \mathrm{~h}^{-2}$. It should be noted that the reference data used in that study was not corrected to account for the neutral species of ionizable compounds (codeine, naproxen and nicotine). If doing that, the $\log \mathrm{K}_{\mathrm{P}_{\text {exp-calc }}}$ mean square deviation would have been even larger $\left(\sim 19 \mathrm{~cm}^{2} \mathrm{~h}^{-2}\right)$.

Alchemical transformations in a gel phase system. It is widely accepted that it is more efficient to perform electrostatic and Lennnard-Jones transformations (coupling/decoupling) in sequence to ensure that the Coulomb interactions are switched off before Lennard-Jones interactions are and vice versa.(29) This avoids overlap of atoms when the attractive Coulomb forces are strong and the repulsive Lennard-Jones forces are turned off or are very weak. However, the skin's barrier structure is in a gel-like state with very low mobility. We have found that the optimal orientation of a permeant molecule might be completely different in a state with its Lennard-Jones interactions turned on and Coulomb interactions turned off, compared to in its fully interacting state, and that the interconversion between the orientations can take very long when the Lennard-Jones interactions are turned on. For example, consider inserting (turning on interactions of) 1-decanol in the sphingoid chains of the skin's barrier structure. If first turning on Lennard-Jones interactions the probability of inserting the molecule with the hydroxyl group oriented away from the head group region, into the interface between the lipid chains, will be approximately the same as inserting it in its most favorable position with the hydroxyl group oriented towards the headgroup region. When turning on the Coulomb interactions it will be unfavourable if the molecule is oriented in the opposite direction and it will take very long for it to turn around. If turning on the Lennard-Jones and the Coulomb interactions simulta- 
bioRxiv preprint doi: https://doi.org/10.1101/2022.02.10.479880; this version posted February 10, 2022. The copyright holder for this preprint (which was not certified by peer review) is the author/funder, who has granted bioRxiv a license to display the preprint in perpetuity. It is made available under aCC-BY-ND 4.0 International license.

a)
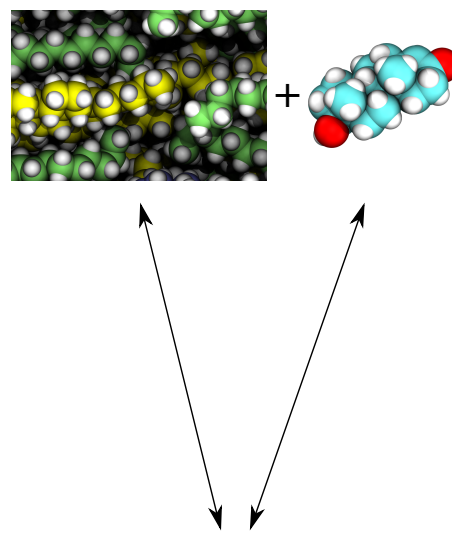

1

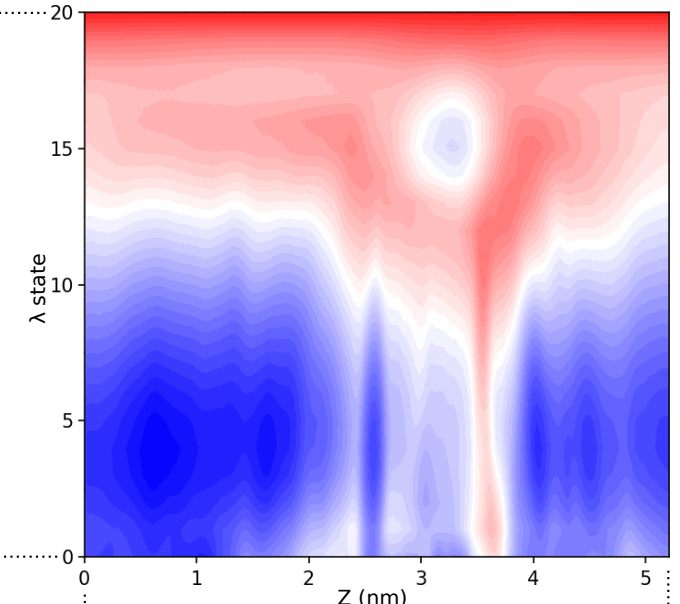

b)
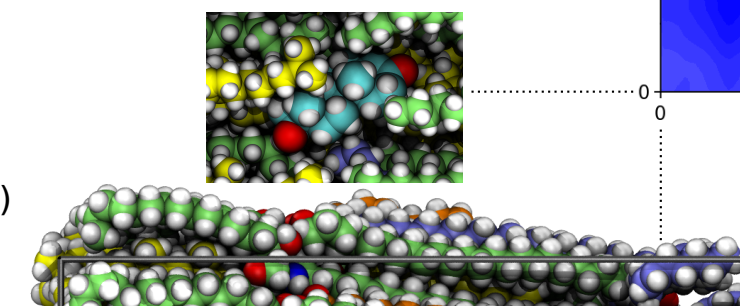

\section{8} 0000090902000 of

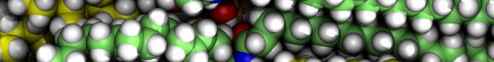

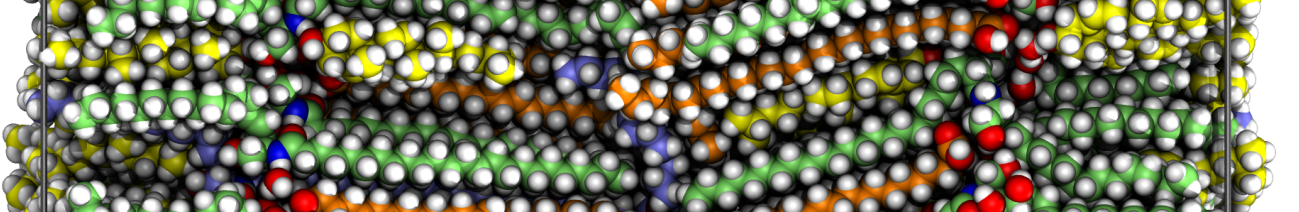

\section{8}

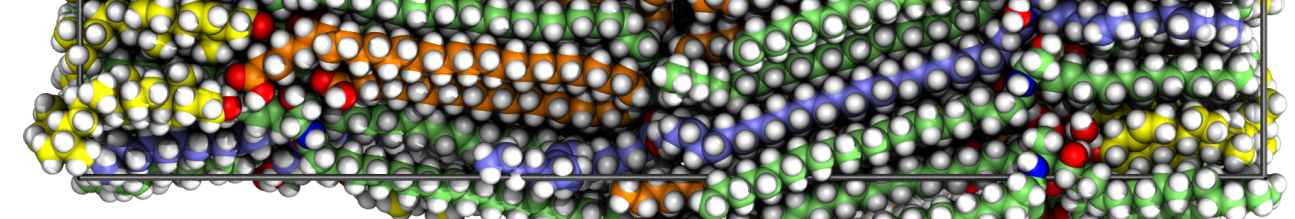

c)
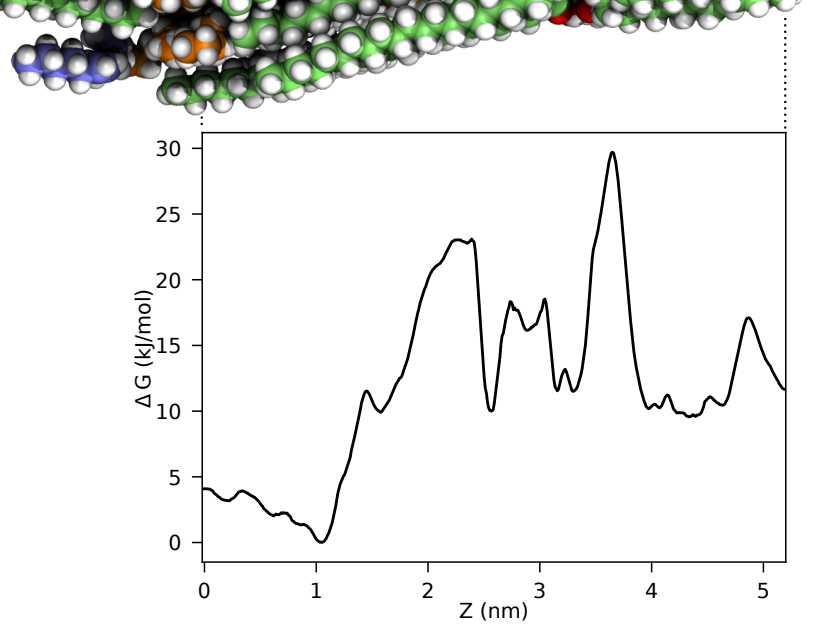

Fig. 2. Calculating the PMF of testosterone through the skin's barrier structure. a) on the right, shows the two-dimensional free energy landscape of testosterone where the alchemical free energy $\lambda$ state is shown on the $Y$ axis -0 is fully interacting and 20 is fully decoupled (as illustrated by the miniatures to the left of the free energy landscape). On the $X$ axis the $Z$ coordinate (in $\mathrm{nm}$ ) through the simulated system is shown. b) shows the skin's barrier system, aligned to illustrate how the PMF is mirrored around 0 (the middle of the system). c) shows the PMF of testosterone through the skin's barrier structure of the fully interacting $\lambda$ state (0 on the $Y$ axis in a). 
neously the electrostatic interactions will favour inserting the molecule correctly from the start.

In this project we decided to use the same, simultaneous interaction transformation, settings for both the twodimensional alchemical free energy/spatial AWH simulations as well as for the one-dimensional AWH alchemical hydration free energy calculations, even if the efficiency might not be optimal for the latter. It should be noted that this problem is at least partially avoided if the alchemical free energy simulations are started from a fully interacting and equilibrated state, or as mentioned above, in a system with fast diffusion in all dimensions.

\section{Methods}

The atomistic model of the human skin's barrier structure presented by Lundborg et al. (6), and originally called $33 / 33 / 33 / 75 / 5 / 0.3^{1}$, was used as the starting structure for the simulations. The starting model had been equilibrated for approximately $270 \mathrm{~ns}, 250 \mathrm{~ns}$ of which were without restraints (6).

All MD simulations in this study (not including the data presented in Figures S1 and S2) were performed using GROMACS 2021 and the second beta version of GROMACS 2022 (30-32), using the Accelerated Weight Histogram (AWH) method for alchemical free energy calculations $(8,25,26)$. A source code modification enabled symmetrizing AWH sampling along a spatial (pulling) reaction coordinate dimension and also changed the number of AWH blocks for autocorrelation analysis from 64 to 128 . These changes are available from the GROMACS gitlab repository (33).

Van der Waals interactions had a cutoff of $1.2 \mathrm{~nm}$ with a smooth force-switch from $1.0 \mathrm{~nm}$ to $1.2 \mathrm{~nm}$. The simulations were run without a dispersion correction for energy and pressure. Coulomb interactions were calculated using PME (34) with a radius of $1.2 \mathrm{~nm}$. Bonds to hydrogen atoms were constrained using the P-LINCS algorithm $(35,36)$. TIP3P (37) parameters were used for water molecules. For the lipid molecules the CHARMM36 lipid force field $(38,39)$ was used. Ceramide parameters were modified to more accurately reproduce the ceramide NP crystal structure.(40) as described in Ref. 6. In order to allow a 3 fs integration time step, hydrogen atoms were made three times heavier by repartitioning the corresponding mass from their bound heavy atoms. Temperature was set to $305.15 \mathrm{~K}$ by using a stochastic dynamics integrator (also referred to as a velocity Langevin dynamics integrator) with a time step of $3 \mathrm{fs}$ and with a time constant $\tau$ of $2 \mathrm{ps}$ (corresponding to a friction constant of $0.5 \mathrm{ps}^{-1}$ ). The pressure was set to $1 \mathrm{~atm}$ and controlled using a stochastic cell rescaling barostat (41) with a time constant of $1.0 \mathrm{ps}$ and a compressibility of $4.5 \times 10^{-5} \mathrm{bar}^{-1}$. In the skin's barrier system the pressure coupling was semi-isotropic with no

\footnotetext{
${ }^{1}$ Relative composition in: molar \% ceramides/molar \% cholesterol/molar $\%$ free fatty acids/relative amount of cholesterol on ceramide sphingoid side/molar \% acyl ceramide EOS (included in the relative ceramide concentration)/water molecules per lipid (not included in the molar \% concentrations of the lipids).
}

compressibility in the $Z$ dimension. For all permeants, at least one simulation was performed with unmodified hydrogen masses and a $2 \mathrm{fs}$ time step in order to verify that the longer ( $3 \mathrm{fs}$ ) time step did not affect the results.

Along the alchemical free energy dimension, 21 equidistantly distributed $\lambda$ states were used for decoupling both van der Waals and Coulomb interactions simultaneously. There were 10 or 100 steps between each sampling of the alchemical free energy $\lambda$-value and 10 samples per update of the $f_{\lambda}$. There was no observable difference between sampling every 10 or 100 steps, but the simulations were faster when sampling less frequently. The estimated initial error was set to $10 \mathrm{~kJ} \mathrm{~mol}^{-1}$. Soft-core transformations (42) with $\alpha=0.5$ and $\sigma=0.3 \mathrm{~nm}$ were applied to both the van der Waals and Coulomb interactions of the solute.

Topologies, i.e. inter- and intramolecular interaction parameters, for all permeants and formulation components except water, were generated using STaGE (43), which in turn uses Open Babel (44) and MATCH (45) to generate GROMACS topologies.

All images representing molecules were prepared using Tachyon (46) in VMD (47).

Hydration Free Energy Calculations. The hydration free energy was calculated starting with a water box large enough to solvate the molecule. The molecule was inserted into the system at a random position with its interactions to the surroundings turned off, as if in vacuum. AWH was used to sample the alchemical free energy $\lambda$ states (8) to calculate the solvation free energy. The initial AWH histogram size, which determines the initial update size of the free energy, was set indirectly by specifying an estimate of the diffusion constant along the alchemical free energy $\lambda$-axis in combination with a rough estimate of the initial error. An input diffusion constant of $1 \times 10^{-3} \mathrm{ps}^{-1}$ was used for the hydration free energy calculations, which means that it is estimated to take approximately $1 \mathrm{~ns}$ to cross the alchemical dimension for one walker. The initial error was set to $10 \mathrm{~kJ} \mathrm{~mol}^{-1} .16$ communicating walkers were run in parallel, with the requirement that only simulations that covered the whole alchemical reaction coordinate counted towards the covering check in the initial AWH stage. The simulations were $60 \mathrm{~ns}$ long per walker for a total simulation time of $960 \mathrm{~ns}$ per solute. For a more detailed description of alchemical hydration free energy calculations using AWH see Lundborg et al. (8).

Permeability Coefficient Calculations. The permeability coefficient, $K_{P}$, and the permeation resistance, $R$, were calculated as follows(48):

$$
\frac{1}{K_{P}}=R=\int_{z_{1}}^{z_{2}} \frac{e^{\beta \Delta G(z)}}{D(z)} d z
$$

where $\Delta G$ is the difference in free energy, compared to the reference state, and $D$ is the local diffusion coefficient, across the skin's barrier structure $(d z)$.

As mentioned above, we assumed a two-step process for calculating the permeability coefficient. The PMF of the first 
layer, out of 80 , was calibrated relative to the hydration free energy of the permeant, whereas the PMF of the remaining 79 layers was calibrated to set the minimum $\Delta G$ to 0 . The permeation resistance, $R$, through the 79 layers was obtained by multiplying $R$ through one layer by a factor 79. The permeation resistances from the two steps were summed in order to obtain the permeability coefficient through the system. The resulting permeability coefficient calculation was:

$$
\begin{aligned}
& \frac{1}{K_{P}}= R=\int_{z_{1}}^{z_{2}} \frac{e^{\beta \Delta G_{\text {rel.water }}(z)}}{D(z)} d z+ \\
& 79 \times \int_{z_{1}}^{z_{2}} \frac{e^{\beta \Delta G_{z e r o e d}(z)}}{D(z)} d z,
\end{aligned}
$$

where $\Delta G_{\text {rel.water }}$ is the free energy relative to the hydration free energy and $\Delta G_{z e r o e d}$ is the free energy shifted so that the PMF minimum is 0 .

The AWH simulation estimates a friction metric tensor $g(z)$ (49), which can be used to derive the diffusion coefficient across the barrier structure, $D(z)=g^{-1}(z)$. To reduce the noise of the local diffusion coefficient curves, an $0.2 \mathrm{~nm}$ wide rolling median filter was applied. When symmetrizing the sampling, along the spatial dimension, by using the absolute coordinate values and accounting for the AWH bias across the sampling boundaries there are usually artificial spikes at the edges of the PMFs. These were been removed by setting the two lowest $(0.005 \mathrm{~nm}$ and $0.015 \mathrm{~nm})$ and highest $(5.200 \mathrm{~nm}$ and $5.210 \mathrm{~nm})$ points in the PMF to the value of their neighbours $(0.025 \mathrm{~nm}$ and $5.19 \mathrm{~nm}$, respectively). These minor adjustments had no effects on the calculated permeability coefficients.

The free energy profile through the skin's barrier structure was calculated using a two-dimensional AWH setup, using a harmonic potential to steer the permeant across the system, also referred to as the $Z$ dimension, and an alchemical free energy reaction coordinate(8). This allows sampling the free energy along the permeation direction and also the relative insertion free energy of the permeant from vacuum. In turn, this enables a direct calibration to the hydration free energy, since the vacuum state is the same in both cases. Like when calculating the hydration free energy, the estimated AWH initial error was set to $10 \mathrm{~kJ} \mathrm{~mol}^{-1}$. The AWH input diffusion constant was set to $3 \times 10^{-5} \mathrm{~nm}^{2} \mathrm{ps}^{-1}$ for the spatial pulling dimension and $5 \times 10^{-5} \mathrm{ps}^{-1}$ along the alchemical free energy dimension. The input diffusion constant affects only the AWH histogram size, it does not affect the computed diffusion coefficient, which is obtained from the AWH friction metric during data analysis. The AWH force constant along the spatial $Z$ dimension (normal to the lamellar stack), steering the permeant relative to the ceramide fatty acid chains using a harmonic pull potential, was set to $25000 \mathrm{~kJ} \mathrm{~mol}^{-1} \mathrm{~nm}^{-2}$. The force constant also determines the resolution along the reaction coordinate dimension. For each permeant, four to six (see Table S1) sets of simulations were run with heavy hydrogen atoms (see above) and a 3 fs integration time step. These were run using 24 communicating walkers, each running for $450 \mathrm{~ns}$. The covering check in the initial AWH stage only took into account simulations

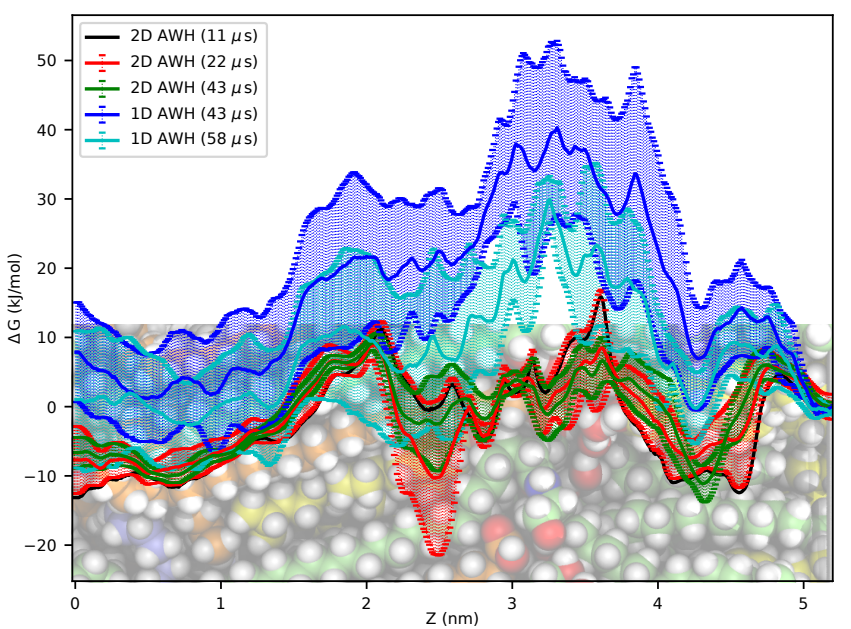

Fig. 3. PMFs of testosterone comparing the $2 \mathrm{D}$ spatial/alchemical $\mathrm{AWH}$ (in the fully interacting alchemical $\lambda$ state) results to $1 \mathrm{D}$ AWH with only a spatial reaction coordinate dimension. In both cases the PMFs are symmetrized and only half the PMFs are presented and the PMFs are calibrated to 0 at the ceramide sphingoid chain interface $(5.2 \mathrm{~nm})$, to make comparisons easier. The uncertainties represent one standard error of the mean. The $11 \mu \mathrm{s} 2 \mathrm{D}$ AWH results are from one set of 24 communicating walkers. AWH analyses do not give a reliable error estimate from one set of simulations, therefore there is no error presented for the $11 \mu \mathrm{s} A W H$ plot (in black). The 1D AWH simulations of $58 \mu$ s required approximately the same computation time as $22 \mu \mathrm{s}$ of 2D AWH. A snapshot of the molecular system is shown at the lower portion of the plot to indicate where the head groups are located (at $\approx 3.2 \mathrm{~nm}$ to $3.3 \mathrm{~nm}$ ).

that covered the whole alchemical dimension and at least a diameter of $0.8 \mathrm{~nm}$ along the spatial dimension. From these simulations a combined diffusion coefficient was calculated using the AWH friction metric from all contributing walkers. The combined PMF was derived from the average of the independent PMFs from the four to six sets of simulations. The combined outputs were used to calculate the permeability coefficients presented in Table S1.

Along the alchemical free energy dimension it is the end states, i.e., the fully interacting and fully decoupled states, that are of highest interest, as the difference in free energy between them corresponds to the probability of transferring the permeant from vacuum into the skin's barrier structure. Therefore, the target distribution used in these simulations put more weight on the end states, especially the state with interactions fully turned on. The target distribution along the alchemical free energy dimension that was used is shown in Fig. S4. Along the spatial pulling dimension the target distribution was uniform.

One-dimensional AWH simulations of testosterone across the barrier structure were also performed, for evaluation and comparison to the two-dimensional AWH results. They were run using four independent sets of simulations, each using 24 communicating AWH walkers running for $600 \mathrm{~ns}$. The simulation settings were the same as specified above, except that there was no alchemical free energy dimension.

\section{Results}

Permeability Coefficient Calculations. Running onedimensional AWH simulations, with only a spatial reaction coordinate dimension across the barrier structure, did 


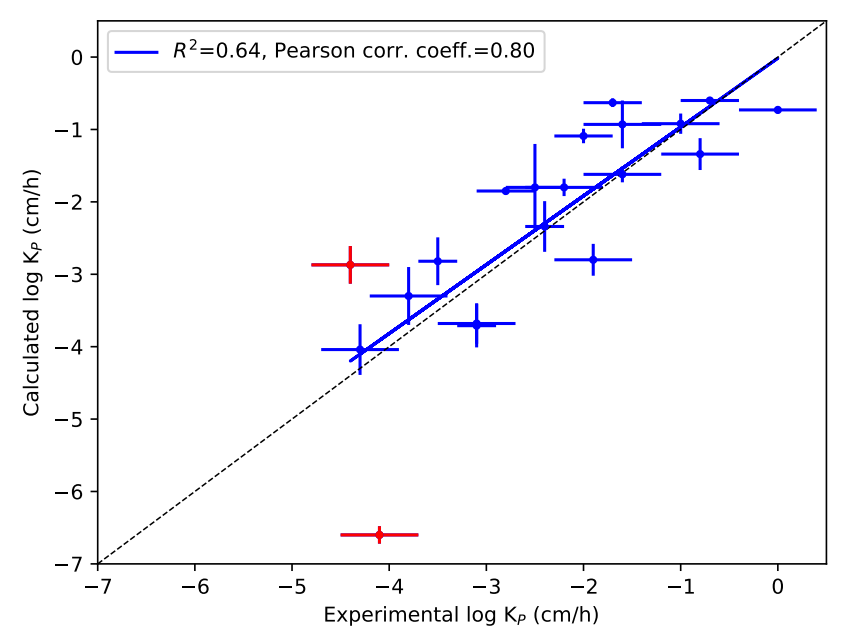

Fig. 4. The correlation between experimental log $K_{P}$ and those calculated from MD simulations. The blue line shows the linear regression, whereas the dashed black line is the identity line. The two largest outliers, urea and hydromorphone, are marked in red (still included in the linear regression). The error bars represent 1 SEM (standard error of the mean), which is approximated for experimental values. Details about the approximation is presented in the header of Table S1.

not sample the free energy profile of testosterone efficiently. None out of four sets of simulations, each consisting of 24 communicating walkers sharing the same AWH bias and running for $600 \mathrm{~ns}$ for a total simulation time of $14.4 \mu \mathrm{s}$ in each of the four independent simulation sets, sampled enough to cover the reaction coordinate with the condition that a single walker must cover a diameter of $0.8 \mathrm{~nm}$ in order to be taken into account. It is possible that this requirement was too strict for a system with inherently slow diffusion. This meant that the one-dimensional AWH simulations did not leave the AWH histogram equilibration stage, as part of the AWH initial stage. Increasing the AWH input diffusion constant would have increased the bias more quickly, meaning covering of the reaction coordinate would have been quicker. However, that might also have resulted in too high free energy barriers in the PMF. The average PMF from the four sets of 1D AWH simulations is compared to the results from the 2D AWH simulations, including an alchemical dimension, in Fig. 3. The results from $58 \mu$ s of $1 \mathrm{D}$ AWH were worse, assuming that the $55 \mu \mathrm{s}$ of $2 \mathrm{D}$ AWH simulations are closest to convergence, than after $11 \mu \mathrm{s}$ of $2 \mathrm{D}$ AWH with an alchemical reaction coordinate, and fairly similar to the FR pulling simulations shown in Fig. S3, but with higher uncertainty, i.e. a larger difference between the four sets of simulations.

The results from the two-dimensional AWH simulations agreed well with the experimental data, thanks to the improved sampling of the free energy landscape. The relationship between calculated and experimental permeability coefficients are presented in Fig. 4. The numerical results are presented in Table S1. The largest outlier was urea, followed by hydromorphone with $\log \mathrm{K}_{\mathrm{P}}$ deviations of -2.4 and $1.5 \mathrm{~cm} \mathrm{~h}^{-1}$, respectively. The average absolute $\log \mathrm{K}_{\mathrm{P}}$ deviation was $0.8 \mathrm{~cm} \mathrm{~h}^{-1}$ (see Table S1). If excluding urea and hydromorphone it would be as low as $0.4 \mathrm{~cm} \mathrm{~h}^{-1}$.

In the supplementary material (Figs. S5 and S6) the PMFs and local diffusion coefficients across the skin's barrier struc- ture are shown. There are also comparisons to our previously published results (7) using FR pulling.

Convergence of Diffusion Coefficients and PMFs. We have observed that the calculated diffusion coefficient depends on the simulation time, with lower diffusion coefficient the longer the simulation time. In general, the same trend is also observed regarding the PMF, i.e., lower free energy barriers with more sampling. We have seen this using all MD simulation sampling methods mentioned herein: AWH (1D with a spatial dimension and 2D with an added alchemical free energy perturbation dimension), umbrella sampling and FR pulling. Most simulations in this study were run for $450 \mathrm{~ns}$ (with 24 communicating AWH walkers). Our observations are that the calculated permeability coefficients are largely unaffected when increasing the simulation time by a factor two, from $450 \mathrm{~ns}$ to $900 \mathrm{~ns}$ per AWH walker (see Table S2 and Fig. S9).

\section{Discussion}

When comparing PMFs of testosterone from the twodimensional AWH simulations performed in this study to previously performed (unpublished) FR pulling simulations (Fig. S3), it is clear that the PMFs from the $30 \mu$ s pulling simulations are closer to the PMFs from AWH. The calculated $\log \mathrm{K}_{\mathrm{P}}$ for testosterone from the FR pulling simulations (taking the 80 stacked lipid bilayers with the same two-step process as described above when calculating the permeability coefficient) was $-4.6 \mathrm{~cm} \mathrm{~h}^{-1}$, compared to $-1.8 \mathrm{~cm} \mathrm{~h}^{-1}$ for the AWH simulations and $-2.5 \mathrm{~cm} \mathrm{~h}^{-1}$ for the ex vivo/in vitro measurements (with reports ranging from $-1.9 \mathrm{~cm} \mathrm{~h}^{-1}$ to $-3.2 \mathrm{~cm} \mathrm{~h}^{-1}(50-53)$, corrected to a temperature of $305 \mathrm{~K}$ ).

The two-dimensional AWH sampling, with one alchemical dimension, was also more efficient than using a onedimensional approach with only a spatial reaction coordinate, as shown in Fig. 3. The main drawback with an alchemical free energy reaction coordinate dimension is that the simulation throughput is lower compared to FR pulling simulations as well as one-dimensional AWH simulations using only a spatial coordinate. However, the possibility to more accurately sample the free energy landscape makes it attractive for problems that are difficult to address with conventional methods, such as permeability coefficient predictions in gelphase systems, like the skin's barrier structure.

Another advantage is that it is easier to prepare permeability calculation simulations using an alchemical dimension than with conventional procedures, as the permeant molecule can be inserted anywhere in the system with all interactions turned off. The AWH simulations will then turn on the interactions where favourable. On the contrary, with standard FR pulling, as well as with one-dimensional AWH pulling or umbrella simulations, the molecule must be grown into the system and/or pulled to suitable starting positions, followed by equilibration of the simulation system, before the production simulations are started. To determine the free energy landscape without an alchemical dimension in the calculation, it 
would also be necessary to know the free energy of inserting the molecular for at least one position along the barrier structure; otherwise the PMF cannot be calibrated compared to a solution or formulation.

The permeability coefficient calculations presented herein only account for the passage of a chemical compound across the skin's barrier structure. Lateral diffusion within the skin's barrier structure is not calculated, and permeation through the stratum corneum cell interiors, as well as through viable cell layers of epidermis, are ignored. While these are admittedly approximations, the contributions from these factors are expected to be negligible in comparison to the permeation resistance across the skin's barrier structure for all but very lipophilic compounds.(54) Second, it is foremostly the permeability across the skin's barrier structure that can be tuned using chemical permeation enhancers, and thereby exploited in transdermal drug delivery design. The two-step process to calculate the permeability coefficient, with partitioning from the delivery vehicle into the lipid barrier followed by permeation through the remaining layers of the lipid barrier, is a simplification of the process, but has given good agreement with experimental measurements. In practice, it only affects the calculated permeability of hydrophilic permeants, when using water as the delivery vehicle, compared to setting the PMF minimum to 0 for all permeation barrier layers.

As can be inferred from Wang and Klauda (22) and from Scheuplein and Blank (54) as well as from Eq. 20 in Marrink and Berendsen (48), and discussed in the Introduction section above, the permeability coefficient does not correspond to an average permeation speed through the skin, but will become lower the thicker the skin sample. Therefore, the permeability coefficient through the skin's barrier structure alone would be higher than through full thickness skin samples used in $e x$ vivo/in vitro permeability measurements. We would therefore expect calculated permeability coefficients, referring exclusively to the skin's barrier structure, to be slightly higher than those measured ex vivo/in vitro through full thickness skin, assuming that the skin samples are intact. Our calculated $\log \mathrm{K}_{\mathrm{P}}$ is $0.1 \mathrm{~cm} \mathrm{~h}^{-1}$ higher than experimental results on average, and $0.2 \mathrm{~cm} \mathrm{~h}^{-1}$ higher if excluding urea and hydromorphone - the largest outliers.

Measuring skin permeability using diffusion cells, such as Franz cells, require long experiment times. How long depends on the permeant's lag time.(55) It is common to run experiments for $24 \mathrm{~h}$ to $72 \mathrm{~h}$.(55) In extreme cases, with low permeability and long lag times, this might even be insufficient. However, long-time exposure to water leads to skin degradation and increased skin permeability. $(55,56)$ These factors can make it difficult to compare theoretical results with those obtained ex vivo/in vitro, since the ex vivo/in vitro measurements may sometimes be too short for the permeation lag time and too long for keeping the skin sample intact. The largest outliers in this study (see Table S1) are urea (log $\mathrm{K}_{\mathrm{P}}$ diff. $-2.5 \mathrm{~cm} \mathrm{~h}^{-1}$ ) and hydromorphone (log $\mathrm{K}_{\mathrm{P}}$ diff. $1.5 \mathrm{~cm} \mathrm{~h}^{-1}$ ), followed by salicylic acid and ethanol. The correlation plot of the results, excluding urea and hydromorphone, is shown in Fig. S7. The permeation enhancing effects of urea (57) were not taken into account in these simulations, which could be a reason for the discrepancy. For hydromorphone, there is a remarkable similarity between the average of three different predicted $\log \mathrm{K}_{\mathrm{P}}$ obtained using mathematical QSPR models of $-3.2 \mathrm{~cm} \mathrm{~h}^{-1}(10,58-60)$ and our predicted $\log \mathrm{K}_{\mathrm{P}}$ at $32{ }^{\circ} \mathrm{C}$ of $-2.9 \mathrm{~cm} \mathrm{~h}^{-1}$, contrasting with the experimentally obtained value of $-4.8 \mathrm{~cm} \mathrm{~h}^{-1}$ at $37^{\circ} \mathrm{C}(61)$, with no corrections for $\mathrm{pH} / \mathrm{pK}_{\mathrm{a}}$. This discrepancy between predicted and experimental data may call for reinvestigation of the experimentally measured permeability coefficient of hydromorphone, preferably from multiple laboratories, although it could of course also indicate permeation properties not picked up by QSPR models either.

\section{Conclusion}

We have shown that the model of the skin's barrier structure that was proposed a few years ago (6) can be used to accurately predict permeability coefficients of a large spectrum of molecules, with clearly better computational efficiency than previous methods.

To our knowledge, this is the first report describing the use of a two-dimensional reaction coordinate with one spatial dimension and one alchemical free energy dimension. It is also the first study to employ the Accelerated Weight Histogram method to sample such a free energy landscape. The obtained PMFs are detailed and some of their features only hinted at when using forward-reverse pulling simulations with very slow pulling speeds. When taking the multiple bilayers of the skin's barrier structure into account, our previously published calculations underestimated the skin permeability coefficients (7), largely explained by the inability of the forward-reverse pulling method to properly sample the free energy landscape.

Experimental permeability coefficient measurements are associated with variations between laboratories as well as with inter- and intraindividual differences. This makes it treacherous to strictly compare calculated permeability coefficients with experimental data. This needs, particularly, to be taken into account when creating QSPR models.(4) A suggested approach for building a reliable QSPR model would therefore be that either all training data should come from one laboratory using the same experimental setup for all compounds or, preferably, there should be measurements from several laboratories, using the same methods, for each compound. When predicting permeability coefficients using MD simulation there is no need for training the method with experimental data, but reliable experimental measurements are required for verifying the techniques and simulation results. The correlation between calculated and experimental permeability coefficients presented here proves the usefulness of MD simulation for transdermal drug delivery design, although the method's precision may presently not always allow for differentiation of very similar molecules.

Perhaps the most important for transdermal drug delivery design is that MD simulation can explain experimental $e x$ vivo/in vitro data by predicting where in the skin's barrier structure the main permeation barriers are located for each 
bioRxiv preprint doi: https://doi.org/10.1101/2022.02.10.479880; this version posted February 10, 2022. The copyright holder for this preprint (which was not certified by peer review) is the author/funder, who has granted bioRxiv a license to display the preprint in perpetuity. It is made available under aCC-BY-ND 4.0 International license.

specific permeant. This knowledge, along with the PMFs (in effect partition profiles) of chemical permeation enhancers, can help selecting suitable drug delivery formulation excipient combinations tailored for each specific API.

\section{ACKNOWLEDGEMENTS}

The computations were enabled by resources provided by the Swedish National Infrastructure for Computing (SNIC 2020/5-220 and 2021/5-217) at PDC Centre for High Performance Computing at KTH and NSC at Linköping University as well as internal resources from ERCO Pharma AB and Erik Lindahl's research group.

\section{DATA AVAILABILITY}

The input data and parameters are available for download from https://doi.org/10.5281/zenodo.5883411

\section{AUTHOR CONTRIBUTIONS}

Lundborg, Norlén, Wennberg and Lindahl designed the research. Lundborg, Lidmar, Hess and Wennberg developed the simulation protocols. Lundborg carried out all simulations and analyzed the data with assistance from Wennberg. All authors wrote the article, with Lundborg being the primary author.

\section{COMPETING FINANCIAL INTERESTS}

\section{This work was funded by ERCO Pharma AB.}

ERCO Pharma $A B$ has submitted a patent application covering using this lipid barrier model for skin permeability calculations (application number PCT/EP2017/076237 and title "SKIN PERMEABILITY PREDICTION").

Norlén, Lundborg and Wennberg have stock options in ERCO Pharma AB. Lundborg and Wennberg are employed by ERCO Pharma AB.

\section{Bibliography}

1. Hiroaki Todo. Transdermal Permeation of Drugs in Various Animal Species. Pharmaceutics, 9(4):33, September 2017. ISSN 1999-4923. doi: 10.3390/pharmaceutics9030033.

2. Harrie Buist, Peter Craig, lan Dewhurst, Susanne Hougaard Bennekou, Carsten Kneuer, Kyriaki Machera, Christina Pieper, Daniele Court Marques, Gilles Guillot, Federica Ruffo, and Arianna Chiusolo. Guidance on dermal absorption. EFSA Journal, 15(6):4873, June 2017. doi: 10.2903/j.efsa.2017.4873.

3. Eui Chang Jung and Howard I. Maibach. Animal models for percutaneous absorption: Animal models for percutaneous absorption. J. Appl. Toxicol., 35(1):1-10, January 2015. ISSN 0260437X. doi: 10.1002/jat.3004.

4. I. Tuncer Degim, W. John Pugh, and Jonathan Hadgraft. Skin permeability data: anomalous results. Int. J. Pharm., 170(1):129-133, August 1998. ISSN 0378-5173. doi: 10.1016/ S0378-5173(98)00113-6.

5. Beatrice Pecoraro, Marco Tutone, Ewelina Hoffman, Victoria Hutter, Anna Maria Almerico, and Matthew Traynor. Predicting Skin Permeability by Means of Computational Approaches: Reliability and Caveats in Pharmaceutical Studies. J. Chem. Inf. Model., 59(5):1759-1771, May 2019. ISSN 1549-9596, 1549-960X. doi: 10.1021/acs.jcim.8b00934.

6. Magnus Lundborg, Ali Narangifard, Christian L. Wennberg, Erik Lindahl, Bertil Daneholt, and Lars Norlén. Human skin barrier structure and function analyzed by cryo-EM and molecular dynamics simulation. J. Struct. Biol., 203(2):149-161, August 2018. ISSN 1047-8477. doi: $10.1016 /$ j.jsb.2018.04.005.

7. Magnus Lundborg, Christian L. Wennberg, Ali Narangifard, Erik Lindahl, and Lars Norlén. Predicting drug permeability through skin using molecular dynamics simulation. J. Control. Release, 283:269-279, August 2018. ISSN 0168-3659. doi: 10.1016/j.jconrel.2018.05.026.

8. Magnus Lundborg, Jack Lidmar, and Berk Hess. The accelerated weight histogram method for alchemical free energy calculations. J. Chem. Phys., 154(20):204103, May 2021. ISSN 0021-9606, 1089-7690. doi: 10.1063/5.0044352.

9. Andrea Rizzi, Travis Jensen, David R. Slochower, Matteo Aldeghi, Vytautas Gapsys, Dimitris Ntekoumes, Stefano Bosisio, Michail Papadourakis, Niel M. Henriksen, Bert L. de Groot Zoe Cournia, Alex Dickson, Julien Michel, Michael K. Gilson, Michael R. Shirts, David L. Mobley, and John D. Chodera. The SAMPL6 SAMPLing challenge: assessing the reliability and efficiency of binding free energy calculations. J. Comput. Aided Mol. Des., 34(5): 601-633, May 2020. ISSN 0920-654X, 1573-4951. doi: 10.1007/s10822-020-00290-5.

10. Russell O. Potts and Richard H. Guy. Predicting Skin Permeability. Pharmaceutical Research, 9(5):663-669, May 1992. ISSN 0724-8741, 1573-904X. doi: 10.1023/A: 1015810312465

11. Michael H. Abraham and Filomena Martins. Human skin permeation and partition: General linear free-energy relationship analyses. J. Pharm. Sci., 93(6):1508-1523, June 2004. ISSN 1520-6017. doi: 10.1002/jps.20070.

12. Ivanka Tsakovska, Ilza Pajeva, Merilin Al Sharif, Petko Alov, Elena Fioravanzo, Simona Kovarich, Andrew P. Worth, Andrea-Nicole Richarz, Chihae Yang, Aleksandra MostragSzlichtyng, and Mark T.D. Cronin. Quantitative structure-skin permeability relationships. Toxicology, 387:27-42, July 2017. ISSN 0300483X. doi: 10.1016/j.tox.2017.06.008.

13. W J Pugh, R Wong, F Falson, B B Michniak, and G P Moss. Discriminant analysis as a tool to identify compounds with potential as transdermal enhancers. J. Pharm. Pharmacol., 57 (11):1389-1396, 2005. ISSN 0022-3573, 2042-7158. doi: 10.1211/jpp.57.11.0003.

14. W. John Albery and Jonathan Hadgraft. Percutaneous absorption: in vivo experiments J. Pharm. Pharmacol., 31(1):140-147, September 1979. ISSN 00223573. doi: 10.1111/j. 2042-7158.1979.tb13456.x.

15. Seung Hun Lee, Se Kyoo Jeong, and Sung Ku Ahn. An Update of the Defensive Barrier Function of Skin. Yonsei Med. J., 47(3):293, 2006. ISSN 0513-5796. doi: 10.3349/ymj.2006. 47.3.293.

16. Arne Naegel, Steffi Hansen, Dirk Neumann, Claus-Michael Lehr, Ulrich F. Schaefer, Gabriel Wittum, and Michael Heisig. In-silico model of skin penetration based on experimentally determined input parameters. Part II: Mathematical modelling of in-vitro diffusion experiments. Identification of critical input parameters. Eur. J. Pharm. Biopharm., 68(2):368-379, February 2008. ISSN 09396411. doi: 10.1016/j.ejpb.2007.05.018.

17. Dominik Selzer, Dirk Neumann, and Ulrich F Schaefer. Mathematical models for dermal drug absorption. Expert Opinion on Drug Metabolism \& Toxicology, 11(10):1567-1583, October 2015. ISSN 1742-5255, 1744-7607. doi: 10.1517/17425255.2015.1063615.

18. Gavin E. Crooks. Entropy production fluctuation theorem and the nonequilibrium work relation for free energy differences. Phys. Rev. E, 60(3):2721-2726, September 1999. doi: 10.1103/PhysRevE.60.2721.

19. Gavin E. Crooks. Path-ensemble averages in systems driven far from equilibrium. Phys. Rev. E, 61(3):2361-2366, March 2000. doi: 10.1103/PhysRevE.61.2361.

20. Ioan Kosztin, Bogdan Barz, and Lorant Janosi. Calculating potentials of mean force and diffusion coefficients from nonequilibrium processes without Jarzynski's equality. J. Chem. Phys., 124(6):064106, February 2006. ISSN 0021-9606, 1089-7690. doi: 10.1063/1. 2166379.

21. L. Y. Chen. Nonequilibrium fluctuation-dissipation theorem of Brownian dynamics. J. Chem Phys., 129(14):144113, October 2008. ISSN 0021-9606, 1089-7690. doi: 10.1063/1. 2992153.

22. Eric Wang and Jeffery B. Klauda. Molecular Structure of the Long Periodicity Phase in the Stratum Corneum. J. Am. Chem. Soc., 141(42):16930-16943, October 2019. ISSN 0002-7863, 1520-5126. doi: 10.1021/jacs.9b08995.

23. Tsuo-Feng Wang, Gerald B. Kasting, and Johannes M. Nitsche. A Multiphase Microscopic Diffusion Model for Stratum Corneum permeability. I. Formulation, Solution, and Illustrative Results for Representative Compounds. J. Pharm. Sci., 95(3):620-648, March 2006. ISSN 00223549. doi: 10.1002/jps.20509.

24. Eric Wang and Jeffery B. Klauda. Structure and Permeability of Ceramide Bilayers and Multilayers. J. Phys. Chem. B, 123(11):2525-2535, March 2019. ISSN 1520-6106, 15205207. doi: 10.1021/acs.jpcb.9b00037.

25. Jack Lidmar. Improving the efficiency of extended ensemble simulations: The accelerated weight histogram method. Phys. Rev. E, 85(5):056708, May 2012. doi: 10.1103/PhysRevE. 85.056708.

26. V. Lindahl, J. Lidmar, and B. Hess. Accelerated weight histogram method for exploring free energy landscapes. J. Chem. Phys., 141(4):044110, July 2014. ISSN 0021-9606, 10897690. doi: $10.1063 / 1.4890371$.

27. Rakesh Gupta, D. B. Sridhar, and Beena Rai. Molecular Dynamics Simulation Study of Permeation of Molecules through Skin Lipid Bilayer. J. Phys. Chem. B, 120(34):8987-8996, August 2016. ISSN 1520-6106, 1520-5207. doi: 10.1021/acs.jpcb.6b05451.

28. Richard M. Venable, Andreas Krämer, and Richard W. Pastor. Molecular Dynamics Simulations of Membrane Permeability. Chem. Rev., 119(9):5954-5997, May 2019. ISSN 00092665. doi: 10.1021/acs.chemrev.8b00486. Publisher: American Chemical Society.

29. Antonia S. J. S. Mey, Bryce K. Allen, Hannah E. Bruce Macdonald, John D. Chodera, David F. Hahn, Maximilian Kuhn, Julien Michel, David L. Mobley, Levi N. Naden, Samarjeet Prasad, Andrea Rizzi, Jenke Scheen, Michael R. Shirts, Gary Tresadern, and Huafeng Xu. Best Practices for Alchemical Free Energy Calculations [Article v1.0]. LiveCoMS, 2(1): 18378, December 2020. doi: 10.33011/livecoms.2.1.18378.

30. Mark James Abraham, Teemu Murtola, Roland Schulz, Szilárd Páll, Jeremy C. Smith, Berk Hess, and Erik Lindahl. GROMACS: High performance molecular simulations through multilevel parallelism from laptops to supercomputers. SoftwareX, 1-2:19-25, September 2015. ISSN 2352-7110. doi: 10.1016/j.softx.2015.06.001.

31. Erik Lindahl, Mark James Abraham, Berk Hess, and David van der Spoel. GROMACS 2021 Manual, January 2021, https://doi.org/10.5281/zenodo.4457591. https://doi.org/10.5281/zenodo.4457591.

32. Szilárd Páll, Artem Zhmurov, Paul Bauer, Mark Abraham, Magnus Lundborg, Alan Gray, Berk Hess, and Erik Lindahl. Heterogeneous parallelization and acceleration of molecular dynamics simulations in GROMACS. J. Chem. Phys., 153(13):134110, October 2020. ISSN 0021-9606, 1089-7690. doi: 10.1063/5.0018516.

33. GROMACS gitlab: 2022-awhsymm-awhcorrblocks, https://gitlab.com/gromacs/gromacs//tree/2022-awhsymm-awhcorrblocks.

34. Ulrich Essmann, Lalith Perera, Max L. Berkowitz, Tom Darden, Hsing Lee, and Lee G. Pedersen. A smooth particle mesh Ewald method. J. Chem. Phys., 103(19):8577-8593, November 1995. ISSN 00219606. doi: 10.1063/1.470117.

35. Berk Hess, Henk Bekker, Herman J. C. Berendsen, and Johannes G. E. M. Fraaije. LINCS A linear constraint solver for molecular simulations. J. Comput. Chem., 18(12):1463-1472, September 1997. ISSN 1096-987X. doi: 10.1002/(SICI)1096-987X(199709)18:12<1463: AID-JCC4>3.0.CO;2-H.

36. Shuichi Miyamoto and Peter A. Kollman. Settle: An analytical version of the SHAKE and RATTLE algorithm for rigid water models. J. Comput. Chem., 13(8):952-962, October 1992. ISSN 1096-987X. doi: 10.1002/jcc.540130805.

37. William L. Jorgensen, Jayaraman Chandrasekhar, Jeffry D. Madura, Roger W. Impey, and Michael L. Klein. Comparison of simple potential functions for simulating liquid water. J. Chem. Phys., 79(2):926-935, 1983. ISSN 00219606. doi: 10.1063/1.445869.

38. Jeffery B. Klauda, Richard M. Venable, J. Alfredo Freites, Joseph W. O'Connor, Douglas J. Tobias, Carlos Mondragon-Ramirez, Igor Vorobyov, Alexander D. MacKerell, and Richard W. Pastor. Update of the CHARMM All-Atom Additive Force Field for Lipids: Validation on Six Lipid Types. J. Phys. Chem. B, 114(23):7830-7843, June 2010. ISSN 1520-6106. doi: $10.1021 /$ jp101759q.

39. Richard M. Venable, Alexander J. Sodt, Brent Rogaski, Huan Rui, Elizabeth Hatcher, Alexander D. Mackerell Jr., Richard W. Pastor, and Jeffery B. Klauda. CHARMM AllAtom Additive Force Field for Sphingomyelin: Elucidation of Hydrogen Bonding and of Positive Curvature. Biophys. J., 107(1):134-145, July 2014. ISSN 0006-3495. doi: 10.1016/j.bpj.2014.05.034.

40. B. Dahlén and I. Pascher. Molecular arrangements in sphingolipids. Thermotropic phase behaviour of tetracosanoylphytosphingosine. Chem. Phys. Lipids, 24(2):119-133, May 1979. ISSN 0009-3084. doi: 10.1016/0009-3084(79)90082-3.

41. Mattia Bernetti and Giovanni Bussi. Pressure control using stochastic cell rescaling. J. Chem. Phys., 153(11):114107, September 2020. ISSN 1089-7690. doi: 10.1063/5.0020514. 
bioRxiv preprint doi: https://doi.org/10.1101/2022.02.10.479880; this version posted February 10,2022 . The copyright holder for this preprint (which was not certified by peer review) is the author/funder, who has granted bioRxiv a license to display the preprint in perpetuity. It is made available under aCC-BY-ND 4.0 International license.

42. Thomas C. Beutler, Alan E. Mark, René C. van Schaik, Paul R. Gerber, and Wilfred F. van Gunsteren. Avoiding singularities and numerical instabilities in free energy calculations based on molecular simulations. Chem. Phys. Lett., 222(6):529-539, June 1994. ISSN 00092614. doi: 10.1016/0009-2614(94)00397-1.

43. Magnus Lundborg and Erik Lindahl. Automatic GROMACS Topology Generation and Comparisons of Force Fields for Solvation Free Energy Calculations. J. Phys. Chem. B, 119(3): 810-823, October 2014. ISSN 1520-6106. doi: 10.1021/jp505332p.

44. Noel M. O'Boyle, Michael Banck, Craig A. James, Chris Morley, Tim Vandermeersch, and Geoffrey R. Hutchison. Open Babel: An open chemical toolbox. J. Cheminf., 3(1):33, October 2011. ISSN 1758-2946. doi: 10.1186/1758-2946-3-33.

45. Joseph D Yesselman, Daniel J Price, Jennifer L Knight, and Charles L Brooks, 3rd. MATCH: an atom-typing toolset for molecular mechanics force fields. J. Comput. Chem., 33(2):189202, January 2012. ISSN 1096-987X. doi: 10.1002/jcc.21963.

46. John Stone. An Efficient Library for Parallel Ray Tracing and Animation. Master's thesis, University of Missouri-Rolla, April 1998

47. William Humphrey, Andrew Dalke, and Klaus Schulten. VMD: Visual molecular dynamics. J. Mol. Graph., 14(1):33-38, February 1996. ISSN 0263-7855. doi: 10.1016/0263-7855(96) 00018-5.

48. Siewert-Jan Marrink and Herman J. C. Berendsen. Simulation of water transport through a lipid membrane. J. Phys. Chem., 98(15):4155-4168, April 1994. ISSN 0022-3654. doi: $10.1021 / \mathrm{j} 100066 \mathrm{a} 040$

49. Viveca Lindahl, Jack Lidmar, and Berk Hess. Riemann metric approach to optimal sampling of multidimensional free-energy landscapes. Phys. Rev. E, 98:023312, Aug 2018. doi: 10.1103/PhysRevE.98.023312.

50. Samir Mitragotri, David A. Edwards, Daniel Blankschtein, and Robert Langer. A mechanistic study of ultrasonically-enhanced transdermal drug delivery. J. Pharm. Sci., 84(6):697-706, June 1995. ISSN 1520-6017. doi: 10.1002/jps.2600840607.

51. R. J. Scheuplein, I. H. Blank, G. J. Brauner, and Dorothy J. Macfarlane. Percutaneous Absorption of Steroids. J. Invest. Dermatol., 52(1):63-70, January 1969. ISSN 0022-202X. doi: $10.1038 /$ jid.1969.9.

52. An-eX Analytical Services Ltd. Assessment of the permeation of testosterone through human skin in vitro from saturated aqueous solution. Technical Report E05/02/17, 2017.

53. O. Siddiqui, M. S. Roberts, and A. E. Polack. Percutaneous absorption of steroids: relative contributions of epidermal penetration and dermal clearance. J. Pharmacokinet. Biopharm., 17(4):405-424, August 1989. ISSN 0090-466X. doi: 10.1007/BF01061455.

54. Robert J. Scheuplein and Irvin H. Blank. Mechanism of Percutaneous Absorption. Iv. Penetration of Nonelectrolytes (alcohols) from Aqueous Solutions and from Pure Liquids. J. Invest. Dermatol., 60(5):286-296, May 1973. ISSN 0022-202X. doi: 10.1111/1523-1747. ep12723090.

55. Barrie Finnin, Kenneth A. Walters, and Thomas J. Franz. In Vitro Skin Permeation Methodology. In Topical and Transdermal Drug Delivery, pages 85-108. John Wiley \& Sons, Ltd, 2011. ISBN 978-1-118-14050-5. doi: 10.1002/9781118140505.ch5. Section: 5 eprint: https://onlinelibrary.wiley.com/doi/pdf/10.1002/9781118140505.ch5.

56. Don A. van Hal, Els Jeremiasse, Hans E. Junginger, Fer Spies, and Joke A. Bouwstra. Structure of Fully Hydrated Human Stratum Corneum: A Freeze-Fracture Electron Microscopy Study. J. Invest. Dermatol., 106(1):89-95, January 1996. ISSN 0022-202X. doi: 10.1111/1523-1747.ep12328031.

57. H. Trommer and R.H.H. Neubert. Overcoming the Stratum Corneum: The Modulation of Skin Penetration. Skin Pharmacol. Physiol., 19(2):106-121, 2006. ISSN 1660-5527, 16605535. doi: 10.1159/000091978.

58. Guoping Lian, Longjian Chen, and Lujia Han. An evaluation of mathematical models for predicting skin permeability. J. Pharm. Sci., 97(1):584-598, January 2008. ISSN 00223549. doi: $10.1002 /$ jps.21074.

59. Michael H Abraham, Harpreet S Chadha, Filomena Martins, Robert C Mitchell, Michael W Bradbury, and Julie A Gratton. Hydrogen bonding part 46: a review of the correlation and prediction of transport properties by an Ifer method: physicochemical properties, brain penetration and skin permeability. Pestic. Sci., 55(1):78-88, 1999. ISSN 1096-9063. doi: 10.1002/(SICI) 1096-9063(199901)55:1<78::AID-PS853> 3.0.CO;2-7. _eprint: https://onlinelibrary.wiley.com/doi/pdt/10.1002/\%28SICl\%2910969063\%28199901\%2955\%3A1\%3C78\%3A\%3AAID-PS853\%3E3.0.CO\%3B2-7.

60. Samir Mitragotri. A theoretical analysis of permeation of small hydrophobic solutes across the stratum corneum based on Scaled Particle Theory. J. Pharm. Sci., 91(3):744-752, March 2002. ISSN 00223549. doi: 10.1002/jps. 10048.

61. Samir D. Roy and Gordon L. Flynn. Transdermal Delivery of Narcotic Analgesics: Comparative Permeabilities of Narcotic Analgesics Through Human Cadaver Skin. Pharm. Res., 6 (10):825-832, 1989. ISSN 0724-8741, 1573-904X. doi: 10.1023/A:1015944018555. 